\title{
Antioxidant Activity and Phenolic Content of Different Parts of Lotus and Optimization of Extraction Condition using Response Surface Methodology
}

\author{
Jae Young Jang, Jong Hoon Ahn, Yang Hee Jo, Bang Yeon Hwang, and Mi Kyeong Lee* \\ College of Pharmacy, Chungbuk National University, Cheongju, Chungbuk 28160, Republic of Korea
}

\begin{abstract}
Nelumbo nucifera Gaertn. (Nymphaeaceae) is commonly called lotus and its leaves are widely been used as functional ingredients due to its antioxidant activity. For maximum efficacy, optimized extraction condition was established using response surface methodology. The high $F$-values, low $p$-values and insignificant $p$-value for lack-of-fit supported the fitness of the model and yielded the second-order polynomial regression for the antioxidant activity. The optimized extract was obtained by the extraction of $1 \mathrm{~g}$ of lotus leaves with $40 \mathrm{~mL}$ of $50 \% \mathrm{MeOH}$ at $10.0^{\circ} \mathrm{C}$, which exerted $70.1 \%$ antioxidant activity. Close correlation between phenolic content and antioxidant activity suggested phenolic compounds as active constituents of lotus leaves. In addition, comparison of different parts of lotus demonstrated the most potent antioxidant activity of flowers, followed by leaves and roots. Taken together, these results provide useful information about lotus leaves for the development as antioxidant ingredients. In addition, flowers and roots as well as leaves are suggested as good sources for antioxidant activity. Keywords-Nelumbo nucifera, antioxidant, various parts, optimization, phenolic content, response surface methodology
\end{abstract}

\section{Introduction}

Nelumbo nucifera Gaertn. (Nymphaeaceae), commonly called lotus, is a perennial aquatic herb that is widely distributed throughout Eastern Asia. Lotus leaves are used for tea and are commercially available in Korea. Steamed rice wrapped with lotus leaves are also consumed as healthy foods for its diverse activity. Other parts of the lotus such as the flowers, seeds and roots are available and have been used in traditional medicine for a long time. Therefore, lotus is widely cultivated and consumed as food and medicine in Korea. Previous studies have revealed the pharmacological effects of lotus leaves, including antioxidant, ${ }^{1,2}$ anti-diabetic ${ }^{3}$ and anti-obesity. ${ }^{4,5}$ Flavonoids and benzylisoquinoline alkaloids have been reported from lotus leaves. ${ }^{6-8}$ Our previous study reported megastigmanes, flavonoid, alkaloids and flavolignans from the lotus leaves and their inhibitory effect on pancreatic lipase. $^{7}$

Oxidative stress derives from an imbalance between the production of reactive oxygen species and antioxidant defense. It is well known as major contributor to diverse

\footnotetext{
*Author for correspondence

Prof. Mi Kyeong Lee, College of Pharmacy, Chungbuk National

University, Cheongju, Chungbuk 28160, Korea

Tel: +82-43-261-2818; E-mail: mklee@chungbuk.ac.kr
}

diseases such as cancer, inflammation, neurodegenerative diseases and diabetes. ${ }^{9-11}$ It also affects age-related symptoms such as fatigue, skin damage and senescence. Antioxidant defense system in our body such as superoxide dismutase, catalase and glutathione reduces reactive oxygen stress (ROS) protects our body from oxidative stress. Natural products with high antioxidant ingredients are also beneficial for reducing oxidative stress. ${ }^{12-14}$

Lotus is well known for antioxidant effect and used as functional ingredients. ${ }^{1,2,15-17}$ For development of products, optimization of extraction condition is required for maximum efficacy. During extraction, many extraction factors such as extraction solvent, extraction time, extraction temperature and solid-liquid ratios affect the composition of extract as well as its biological activity. ${ }^{18-20}$ Response surface methodology (RSM) is a statistical tool and takes into several factors simultaneously using rationally designed experiment. Therefore, optimal condition can be effectively derived especially in case of several variables. ${ }^{21-23}$

In the present study, antioxidant activity of lotus leaves was investigated using DPPH radical scavenging assay system and optimized extraction condition was derived using RSM with three-level-three-factor Box-Behnken design (BBD). The correlation between activities and phenolic content was also analyzed. Since different plant parts may contain different types of constituents which 
contribute to the biological activities, ${ }^{24-27}$ the antioxidant activities of different parts of lotus were also compared.

\section{Experimental}

Plant material - Leaves, flowers, seeds and roots of $N$. nucifera were prepared from the local herbal market in Chungbuk, Korea in January 2016. They were identified by the herbarium of the College of Pharmacy at Chungbuk National University, where voucher specimens were deposited (CBNU201601-NNL, NNF, NNS and NNR). Leaves, flowers, seeds and roots of lotus were extracted twice with $80 \% \mathrm{MeOH}$ for the evaluation of antioxidant activity and phenolic content.

Optimization using response surface of methodology RSM with three variables and three levels BBD was used to optimize the extraction conditions of lotus leaves for antioxidant activity. In the present study, extraction solvent, $\mathrm{MeOH}\left(X_{1}\right)$, extraction temperature $\left(X_{2}\right)$ and solvent volume / dried lotus leaves $\left(X_{3}\right)$ were chosen as three independent extraction variables. After determination of the ranges of these variables on the basis of a preliminary single factor experiment, complete design with 15 experimental points including three replication of the center points (all variables were coded as zero) was set up.

The fitness of the polynomial model equation to the responses was evaluated with the coefficients of $R^{2}$ and the lack of fit was evaluated using F-test.

Evaluation of antioxidant activity - The antioxidant activity was evaluated by measuring the free radical scavenging activity using 2,2-diphenyl-1-picrylhydrazyl (DPPH). The radical scavenging activity was determined by measuring the absorbance at $517 \mathrm{~nm}$. The relative radical scavenging activity (\%) was calculated as [1absorbance of solution with sample and DPPH / absorbance of solution with DPPH] $\times 100$.

Measurement of Total Phenolic Content - The total phenolic content was measured with a Folin-Ciocalteau assay. Folin-Ciocalteau's phenol reagent was added to the 96-well plate containing the test samples. After $5 \mathrm{~min}$ of incubation with gentle shaking, $7 \% \mathrm{Na}_{2} \mathrm{CO}_{3}$ was added to the reaction mixture. The reaction mixture was left in the dark for $90 \mathrm{~min}$ at room temperature. The absorbance was measured at $630 \mathrm{~nm}$ with a microplate reader. The total phenolic content was expressed as gallic acid equivalent (GAE) using gallic acid as a standard.

\section{Results and Discussion}

We first investigated the antioxidant activity of extract of lotus leaves using DPPH radical scavenging assay. The total extract of lotus leaves showed dose-dependent antioxidant activity with $\mathrm{IC}_{50}$ values of $100.2 \mu \mathrm{g} / \mathrm{mL}$. We previously investigated the constituents of lotus leaves and reported thirty two compounds such as megastigmanes, alkaloids, flavonoids and phenolic compounds. ${ }^{7}$ Therefore, we next investigated the antioxidant activity of the constituents of lotus leaves. Four constituents were selected from each skeleton such as vomifoliol (1), a megastigmane, roemerine- $N$-oxide (2), an alkaloid, quercetin (3), a flavonoid and trans- $N$-feruloyltyramine (4), a phenolic compound (Fig. 1). Among them, quercetin (3) and trans$N$-feruloyltyramine (4) exerted antioxidant activity with $\mathrm{IC}_{50}$ values of 6.6 and $69.4 \mu \mathrm{g} / \mathrm{mL}$, respectively. Vomifoliol (1) and roemerine- $N$-oxide (2), however, showed weak activity with $\mathrm{IC}_{50}$ values of $>100 \mu \mathrm{g} / \mathrm{mL}$. These results suggested that phenolic compounds contribute the antioxidant activity of lotus leaves.

Extraction conditions greatly affect the biological activity as well as the constituents of extract. ${ }^{18-20}$ Therefore, effect of extraction condition of lotus leaves on antioxidant activity and phenolic content was investigated. In addition, extraction conditions of lotus leaves was optimized for maximum antioxidant activity using RSM employing BBD with three-level-three-factor (Table 1).

Extraction solvent, extraction temperature and solvent/ sample ratio were chosen for extraction variables and the ranges of each variables were selected as extraction
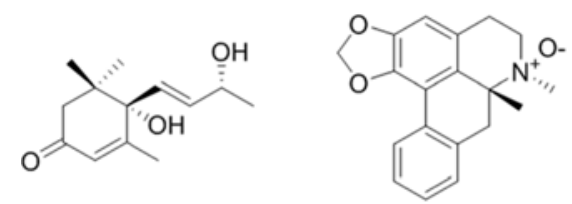

vomifoliol (1) roemerine- $\mathrm{N}$-oxide (2)

$\mid C_{50}:>100 \mu \mathrm{g} / \mathrm{ml}$

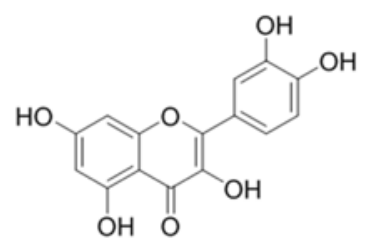

quercetin (3)

$\mid \mathrm{C}_{50}: 6.6 \mu \mathrm{g} / \mathrm{ml}$

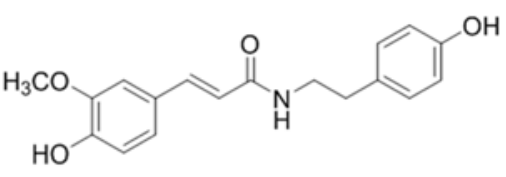

trans- $\mathrm{N}$-feruloyltyramine (4)

${ }^{\prime} C_{50}: 69.4 \mu \mathrm{g} / \mathrm{ml}$

Fig. 1. Structures and antioxidant $\mathrm{IC}_{50}$ values of compounds from lotus leaves. 
Table 1. A Box-Behnken design for independent variables and their responses

\begin{tabular}{|c|c|c|c|c|c|}
\hline \multirow[b]{2}{*}{ Run } & \multicolumn{3}{|c|}{ Actual variables } & \multicolumn{2}{|c|}{ Observed values } \\
\hline & $\begin{array}{c}\mathrm{MeOH} \text { in water } \\
(\%)\end{array}$ & $\begin{array}{c}\text { Extraction } \\
\text { temperature }\left({ }^{\circ} \mathrm{C}\right)\end{array}$ & $\begin{array}{l}\text { Solvent / sample } \\
(\mathrm{mL} / \mathrm{g}) \mathrm{S}\end{array}$ & $\begin{array}{c}\text { Antioxidant activity }{ }^{\mathrm{a}} \\
(\%)\end{array}$ & $\begin{array}{c}\text { Total phenolic } \\
\text { ( } \mu \mathrm{g} \text { GAE / mg extract) }\end{array}$ \\
\hline 1 & 0 & 40 & 10 & 54.3 & 111.58 \\
\hline 2 & 100 & 70 & 30 & 45.3 & 73.93 \\
\hline 3 & 50 & 40 & 30 & 56.7 & 104.91 \\
\hline 4 & 50 & 40 & 30 & 54.9 & 127.07 \\
\hline 5 & 0 & 10 & 30 & 50.8 & 97.60 \\
\hline 6 & 50 & 70 & 50 & 50.9 & 96.73 \\
\hline 7 & 0 & 40 & 50 & 53.1 & 98.24 \\
\hline 8 & 50 & 70 & 10 & 67.8 & 130.73 \\
\hline 9 & 50 & 10 & 10 & 53.3 & 128.36 \\
\hline 10 & 100 & 40 & 10 & 56.9 & 95.87 \\
\hline 11 & 50 & 40 & 30 & 59.8 & 122.12 \\
\hline 12 & 100 & 40 & 50 & 48.9 & 67.26 \\
\hline 13 & 100 & 10 & 30 & 48.7 & 81.89 \\
\hline 14 & 0 & 70 & 30 & 43.4 & 95.66 \\
\hline 15 & 50 & 10 & 50 & 70.4 & 140.41 \\
\hline
\end{tabular}

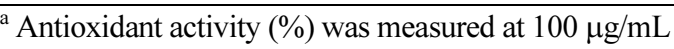

Table 2. Regression coefficients and their significances in the second-order polynomial regression equation

\begin{tabular}{cccrr}
\hline \hline & Coefficient & Standard error & $t$ value & $p$ value \\
\hline Intercept & 53.817 & 1.647 & 32.681 & $<0.001$ \\
$X_{1}$ & -0.223 & 1.008 & -0.221 & 0.834 \\
$X_{2}$ & -1.974 & 1.008 & -1.957 & 0.108 \\
$X_{3}$ & -1.131 & 1.008 & -4.752 & 0.005 \\
$X_{1}^{2}$ & -7.053 & 1.484 & -12.800 & $<0.001$ \\
$X_{2}^{2}$ & 0.294 & 1.484 & 0.198 & 0.851 \\
$X_{3}^{2}$ & 6.450 & 1.484 & 4.378 & 0.007 \\
$X_{1} X_{2}$ & 1.008 & 1.426 & 0.706 & 0.511 \\
$X_{1} X_{3}$ & -1.693 & 1.426 & -1.187 & 0.289 \\
$X_{2} X_{3}$ & -8.510 & 1.426 & -5.967 & 0.002 \\
\hline
\end{tabular}

solvent $\left(X_{1}, \mathrm{MeOH}\right.$ concentration in water, 0,50 and $100 \%)$, extraction temperature $\left(X_{2}, 10,40\right.$ and $\left.70^{\circ} \mathrm{C}\right)$ and solvent/sample ratio $\left(X_{3}, 10,30\right.$ and 50$)$ on the preliminary single factor experiment. Antioxidant activities were varied from 43.4 to $70.4 \%$ depending on of each experimental point, which showed the importance of extraction condition for antioxidant activity.
Second-order polynomial regression equation was established by RSM for the evaluation of the relationship between variables and responses. Greater coefficients with smaller $p$-value $(\mathrm{p}<0.05)$ indicated the considerable effect of these coefficients on response (Table 2). The value of multiple determination $\left(R^{2}\right)$ was 0.946 , which demonstrated effectiveness of this model. Insignificant pvalue of lack of fit, 0.767 also indicated the adaptability of this model to experimental data (Table 3). Relationship between every two variables for antioxidant activity was also shown in three dimensional response surface plots based on regression equations (Fig. 2). Collectively, this model is adequately fitted to the experimental data and suitable for optimization.

Multiple regression analysis on the experimental data yielded the second-order polynomial regression equation for coded values as follows,

Antioxidant activity $(\%)=53.82-0.22 X_{1}-1.98 X_{2}-1.13$ $X_{3}-7.05 X_{1}^{2}+0.29 X_{2}^{2}+6.45 X_{3}^{2}+1.01 X_{1} X_{2}-1.69 X_{1} X_{3}-$ $8.51 X_{2} X_{3}$

Table 3. ANOVA for response surface regression equation

\begin{tabular}{lccccc}
\hline \hline & Sum of square & Degree of freedom & Mean square & $F$ value & $p$ value \\
\hline Regression & 715.28 & 9 & 79.496 & 9.77 & 0.011 \\
Linear & 41.80 & 3 & 13.933 & 1.71 & 0.279 \\
Square & 368.28 & 3 & 122.761 & 15.09 & 0.006 \\
Interaction & 305.20 & 3 & 101.733 & 12.51 & 0.009 \\
Residual error & 40.68 & 5 & 8.135 & 0.41 & 0.767 \\
Lack-of-fit & 15.39 & 3 & 12.130 & & \\
Pure error & 25.29 & 2 & & & \\
Total & 755.96 & 14 & & &
\end{tabular}



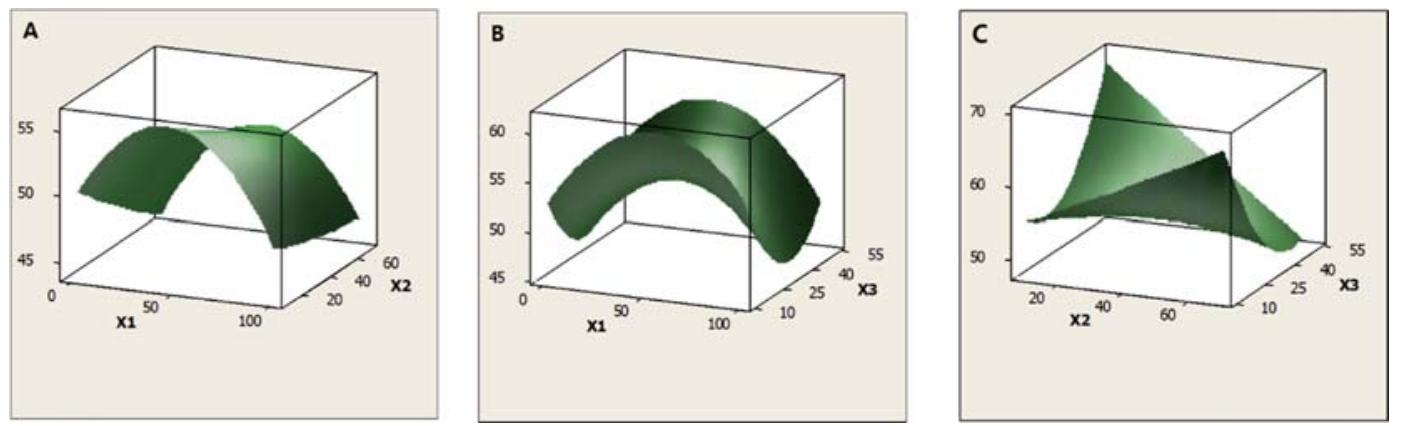

Fig. 2. Response surface plots of antioxidant activity by $\mathrm{MeOH}$ concentration $\left(X_{1}\right)$, extraction temperature $\left(X_{2}\right)$ and solvent/sample ratio $\left(X_{3}\right)$.

Table 4. Predicted and observed values of antioxidant activity under optimized condition

\begin{tabular}{ccccc}
\hline \hline & \multicolumn{2}{c}{ Extraction condition } & \multicolumn{2}{c}{ Antioxidant activity ${ }^{\text {a }}$} \\
\hline $\mathrm{MeOH}$ in water $(\%)$ & Extraction temperature $\left({ }^{\circ} \mathrm{C}\right)$ & Solvent/sample ratio $(\mathrm{mL} / \mathrm{g})$ & Predicted $(\%)$ & Observed $(\%)$ \\
\hline 50.0 & 10.0 & 40.0 & 70.3 & 70.1 \\
\hline
\end{tabular}

${ }^{a}$ Antioxidant activity (\%) was measured at $100 \mu \mathrm{g} / \mathrm{mL}$.

Extraction yield $(\%)=30.40-6.25 X_{1}+4.20 X_{2}+3.35$ $X_{3}-4.70 X_{1}^{2}+1.00 X_{2}^{2}-1.90 X_{3}^{2}+1.50 X_{1} X_{2}-0.20 X_{1} X_{3}+$ $0.50 X_{2} X_{3}$

Among extraction variables, quadratic term $\left(X_{1}^{2}\right)$ of $\mathrm{MeOH}$ concentration showed the most significant effect on antioxidant activity with $p$-value of $<0.001$ (Table 2 ). The linear $\left(X_{3}\right)$ and quadratic term $\left(X_{3}^{2}\right)$ of solvent/sample ratio together with the interaction term of extraction temperature with $p$ values of $0.005,0.007$ and 0.002 , respectively. Other variables, however, did not show any significant effect on antioxidant activity of lotus leaves in our present study.

Three dimensional response surface plots are consistent with multiple regression analysis. Extraction solvent showed dramatic effect on antioxidant activity of lotus leaves. Antioxidant activity of lotus leaves was increased with increasing $\mathrm{MeOH}$ concentration but decreased thereafter, which showed the importance of quadratic term $\left(X_{1}^{2}\right)$ of $\mathrm{MeOH}$ concentration (Figs. 2A and 2B). Similar quadratic pattern of solvent/sample ratio was observed at fixed extraction temperature (Fig. 2B).

Based on our results, an optimization for extraction condition of lotus leaves for maximum antioxidant activity was suggested by RSM and verified by experiment. Optimal extraction condition for maximum antioxidant activity was determined as $\mathrm{MeOH}$ concentration in water, $50.0 \%$, temperature, $10.0^{\circ} \mathrm{C}$, and solvent/sample ratio, 40 , which predicted $70.3 \%$ antioxidant activity (Table 4). Extraction of lotus leaves extract prepared under optimized condition showed $70.1 \%$ antioxidant activity, which was well matched with predicted values.

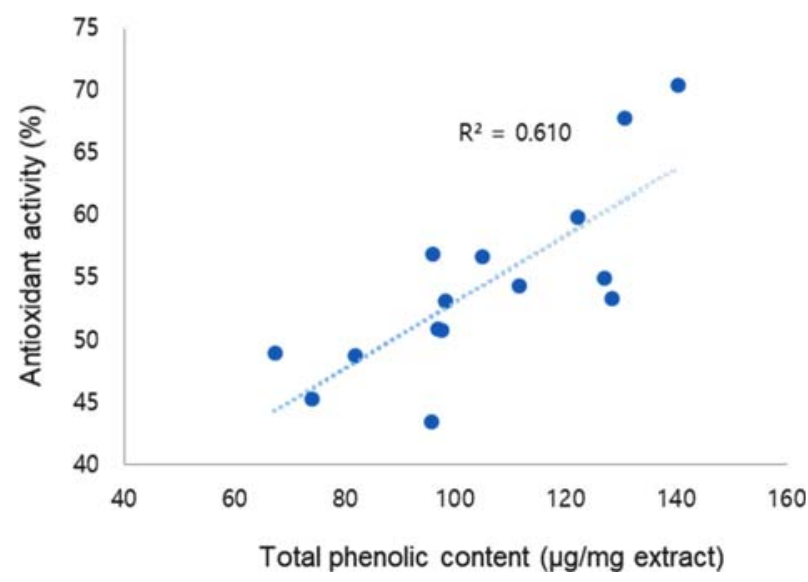

Fig. 3. Correlation between antioxidant activity and phenolic content of the lotus leaves extract from different extraction condition.

Phenolic compounds contribute the antioxidant activity of lotus leaves. Therefore, correlation between antioxidant activity and phenolic content was investigated. The total phenolic content in lotus leaves extract was varied from 67.3 to $140.4 \mu \mathrm{g}$ GAE/mg extract depending on extraction conditions (Table 1) and antioxidant activity was quite proportional to phenolic content with $R^{2}$ value of 0.610 (Fig. 3). These results suggested that antioxidant activity of lotus leaves was achieved by phenolic constituents to some extent, which is also supported by the antioxidant activity of its constituents of quercetin (3) and trans- $N$ feruloyltyramine (4) (Fig. 1).

Although lotus leaves are widely used for food or tea, other parts of the lotus such as the fruit, flowers, seeds and roots are available and have been used in traditional 
Table 5. Antioxidant activity and total phenolic content of the leaves, flowers, seeds and the roots of lotus

\begin{tabular}{ccc}
\hline \hline Parts & $\begin{array}{c}\text { Antioxidant activity }{ }^{\text {a }} \\
(\%)\end{array}$ & $\begin{array}{c}\text { Total phenolic content } \\
\text { (g GAE/mg extract) }\end{array}$ \\
\hline Leaves & 47.8 & 68.9 \\
Flowers & 59.3 & 139.7 \\
Seeds & 42.1 & 32.2 \\
Roots & 21.9 & 6.3 \\
\hline
\end{tabular}

${ }^{a}$ Antioxidant activity (\%) was measured at $100 \mu \mathrm{g} / \mathrm{mL}$.

medicine for a long time. Different parts of plant may contain different types of constituents which contribute to the biological activities. ${ }^{24,27}$ In other aspect, plants use similar biosynthetic pathway among different parts, therefore, they share common structure or substituents which are characteristic to each plant. ${ }^{25,26}$ Therefore, the antioxidant activity of different parts of lotus such as flowers, seeds and roots were also compared.

As shown in Table 5, the flower showed the most potent antioxidant activity followed by leaves, seeds and roots. Due to the importance of phenolic contents for antioxidant activity of lotus, the total phenolic content of each part was measured. The total phenolic content was the highest in flower, followed by leaves, seeds and roots, which showed the correlation with antioxidant activity. These results show that different parts of lotus commonly contains phenolic constituents, however, the amounts and structures are different among different parts. Therefore, other parts also can be used as important antioxidant resources for further development.

In conclusion, lotus leaves are widely used for tea and also consumed as healthy foods for its diverse activity. Therefore, extraction condition was optimized using response surface methodology for maximal efficacy. In addition, constituents and antioxidant activity of other parts of the lotus such as the flowers, seeds and roots have been compared. Our present study presented optimized extraction condition of lotus leaves for maximal antioxidant activity. Among parts of louts, flower showed the most potent antioxidant activity with high phenolic contents. Therefore, our present study will provide efficient extraction condition of lotus leaves for maximal activity and suggested other parts as important antioxidant resources for further development.

\section{Acknowledgement}

This work was supported by Basic Science Research Program (2018R1D1A1A09082316) through the National Research Foundation of Korea.

\section{References}

(1) Zhu, M. Z.; Wu, W.; Jiao, L. L.; Yang, P. F.; Guo, M. Q. Molecules 2005, 20, 10553-10565.

(2) Je, J. Y.; Lee, D. B.; Food Funct. 2015, 6, 1911-1918.

(3) Liao, C. H., Lin, J. Y Food Chem. Toxicol. 2013, 58, 416-422.

(4) Du, H.; You, J. S.; Zhao, X.; Park, J. Y.; Kim, S. H.; Chang, K. J. J. Biomed. Sci. 2010, 17, S1-S42.

(5) Lin. S.; Li, D.; Huang, B.; Chen, Y.; Lu, X.; Wang, Y. J. Ethnopharmacol. 2013, 149, 263-269.

(6) Nakamura, S.; Kasahima, S.; Tanabe, G.; Oda, Y.; Yokota, N.; Fujimoto, K.; Matsumoto, T.; Sakuma, R.; Ohta, T.; Ogawa, K.; Nishida, S.; Miki, H.; Matsuda, H.; Muraoka, O.; Yoshikawa, M. Bioorg. Med. Chem. 2013, 21, 779-787.

(7) Ahn, J. H.; Kim, E. S.; Lee, C.; Kim, S.; Cho, S. H.; Hwang, B. Y.; Lee, M. K. Bioorg. Med. Chem. Lett. 2013, 23, 3604-3608.

(8) Paudel, K. R.; Panth, N. Evid. Based Complement. Alternat. Med. 2015, 2015, 789124

(9) Maritim, A. C.; Sanders, R. A.; Watkins, J. B. 3rd. J. Biochem. Mol. Toxicol. 2003, 17, 24-38 .

(10) Reuter, S.; Gupta, S. C.; Chaturvedi, M. M.; Aggarwal, B. B. Free Radic. Biol. Med. 2010, 49, 1603-1616.

(11) Pohanka, M. Curr. Med. Chem. 2014, 21, 356-364.

(12) Pisoschi, A. M.; Pop, A. Eur. J. Med. Chem. 2015, 97, 55-74

(13) Alasalvar, C.; Bolling, B. W. Br. J. Nutr. 2015, 113, S68- S78

(14) Viapiana, A.; Wesolowski, M. Plant Foods Hum. Nutr. 2017, 72, 82-87.

(15) Park, Y. S.; Towantakawanit, K.; Kowalska, T.; Jung S.T.; Ham, K.S.; Heo, B. G.; Cho, J. Y.; Yun, J. G.; Kim, H. J.; Gorinstein, S. J. Med. Food 2009, 12, 1057-1064.

(16) Zhao, X.; Shen, J.; Chang, K. J.; Kim, S. H. J. Agric. Food Chem. 2014, 62, 6227-6235.

(17) Liu, Y.; Ma, S. S.; Ibrahim, S. A.; Li, E. H.; Yang, H.; Huang, W. Food Chem. 2015, 185, 159-164.

(18) Zhang, W. M.; Huang, W. Y.; Chen, W. X.; Han, L.; Zhang, H. D Molecules 2014, 19, 16416-16427.

(19) Jeong, J. Y.; Jo, Y. H.; Lee, K. Y.; Do, S. G.; Hwang, B. Y.; Lee, M. K. Bioorg. Med. Chem. Lett. 2014, 24, 2329-2333.

(20) Lu, C. L.; Zhu, Y. F.; Hu, M. M.; Wang, D. M.; Xu, X. J.; Lu, C. J.; Zhu, W. Molecules 2015, 20, 625-644.

(21) Ferreira, S. L. C.; Bruns, R. E.; Ferreira, H. S.; Matos, G. D.; David, J. M.; Brandão, G. C.; da Silva, E. G.; Portugal, L. A.; dos Reis, P. S.; Souza, A. S.; dos Santos, W. N. Anal. Chim. Acta. 2007, 597, 179-186.

(22) Bezerra, M. A.; Santelli, R. E.; Oliveira, E. P.; Villar, L. S.; Escaleira, L. A. Talanta 2008, 76, 965-977.

(23) Xu, Q.; Shen, Y.; Wang, H.; Zhang, N.; Xu, S.; Zhang, L. Food Chem. 2013, 138, 2122-2129.

(24) He, Y.; Peng, J.; Hamann, M. T.; West, L. M. J. Nat. Prod. 2014 $77,2138-2143$

(25) Jo, Y. H.; Shin, B.; Liu, Q.; Lee, K. Y.; Oh, D. C.; Hwang, B. Y.; Lee, M. K. J. Nat. Prod. 2014, 77, 2361-2366.

(26) Hiep, N. T.; Kwon, J.; Kim, D. W.; Hwang, B. Y.; Lee, H. J.; Mar, W.; Lee, D. Phytochemistry 2015, 111, 141-148.

(27) Chung, I. M.; Lim, J. J.; Ahn, M. S.; Jeong, H. N.; An, T. J.; Kim, S. H. J. Ginseng Res. 2016, 40, 68-75.
Received June 18, 2018

Revised November 5, 2018 Accepted November 9, 2018 